\title{
Native-like SARS-CoV-2 Spike Glycoprotein Expressed by ChAdOx1 nCoV-19/AZD1222 Vaccine
}

Yasunori Watanabe, Luiza Mendonça, Elizabeth R. Allen, Andrew Howe, Mercede Lee, Joel D. Allen, Himanshi Chawla, David Pulido, Francesca Donnellan, Hannah Davies, Marta Ulaszewska, Sandra Belij-Rammerstorfer, Susan Morris, Anna-Sophia Krebs, Wanwisa Dejnirattisai, Juthathip Mongkolsapaya, Piyada Supasa, Gavin R. Screaton, Catherine M. Green, Teresa Lambe,* Peijun Zhang,* Sarah C. Gilbert,* and Max Crispin*

Cite This: ACS Cent. Sci. 2021, 7, 594-602

Read Online

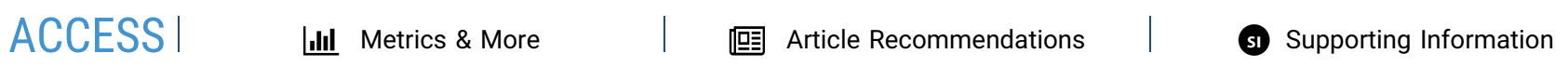

ABSTRACT: Vaccine development against the SARS-CoV-2 virus focuses on the principal target of the neutralizing immune response, the spike (S) glycoprotein. Adenovirus-vectored vaccines offer an effective platform for the delivery of viral antigen, but it is important for the generation of neutralizing antibodies that they produce appropriately processed and assembled viral antigen that mimics that observed on the SARS-CoV-2 virus. Here, we describe the structure, conformation, and glycosylation of the $S$ protein derived from the adenovirus-vectored ChAdOx1 nCoV-19/ AZD1222 vaccine. We demonstrate native-like post-translational processing and assembly, and reveal the expression of $S$ proteins on the surface of cells adopting the trimeric prefusion conformation. The data presented here confirm the use of ChAdOxl adenovirus vectors as a leading platform technology for SARS-CoV-2 vaccines.

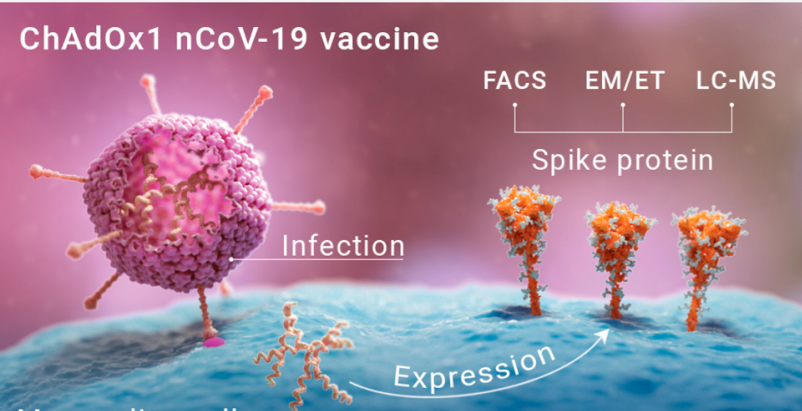

Mammalian cell

\section{INTRODUCTION}

Vaccines against severe acute respiratory syndrome coronavirus-2 (SARS-CoV-2) are an essential countermeasure to stem the COVID-19 pandemic. Vaccine development efforts aim to produce both a strong $\mathrm{T}$ cell response and a neutralizing immune response against the virus, the main target being the spike (S) proteins that protrude from the viral envelope. ${ }^{1}$ The $S$ protein is responsible for mediating host-cell entry, with the S1 and S2 subunits facilitating angiotensin-converting enzyme 2 (ACE2) receptor binding and membrane fusion, respectively. $^{2-4}$ The SARS-CoV-2 S gene encodes the extensively glycosylated trimeric class I viral fusion protein with $22 \mathrm{~N}$ linked glycans per protomer. ${ }^{5}$

While the coronavirus $S$ glycoprotein is the principal target for SARS-CoV-2 vaccine design, leading vaccine candidates and recently licensed vaccines utilize a variety of constructs and strategies. ${ }^{6}$ For example, both Moderna's mRNA-1273 and Pfizer's BNT162b2 $2^{7}$ encode full length $S$ with two mutations to stabilize the prefusion conformation, ${ }^{8}$ and Sinovac's CoronaVac inactivated virus vaccine presents the wild-type $S$ on the viral surface, ${ }^{9}$ although the majority of spikes are in the postfusion conformation. ${ }^{10}$ One key aim for SARS-CoV-2 vaccine development is to elicit a robust immune response against the spike, and more specifically the receptor-binding domain (RBD), where many neutralizing epitopes are located. ${ }^{10-15}$ To this end, many vaccine candidates include (two or more) stabilizing mutations in the $S$ protein, such that the protein maintains the prefusion conformation and avoids shedding of $\mathrm{S} 1$. $^{3}$

The replication-deficient chimpanzee adenovirus-vectored vaccine, ChAdOx1 nCoV-19/AZD1222 (hereafter referred to as ChAdOx1 nCoV-19), encodes the full-length wild-type SARS-CoV-2 spike protein. ChAdOxl nCoV-19 has previously been shown to elicit not only strong neutralizing antibody responses but also robust spike-specific T-cell responses. ${ }^{16-19}$ Although adenovirus-vectored vaccines are a promising way to deliver viral glycoprotein antigens, the processing and presentation of the SARS-CoV-2 spike are yet to be characterized. Understanding the molecular features of the expressed viral antigen is important for the interpretation of the immune response to this vaccine. Here, we determine the

Received: January 18, 2021

Published: April 2, 2021 
A

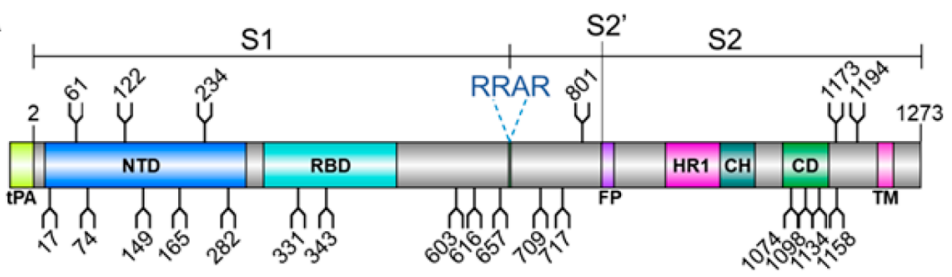

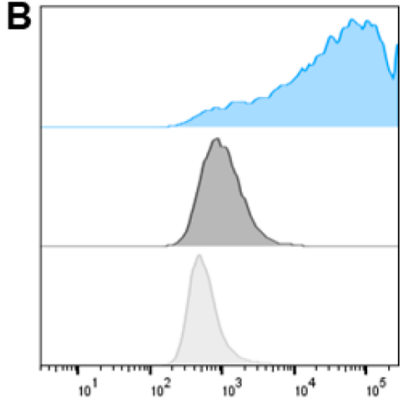

Anti ChAdOx1 nCov-19

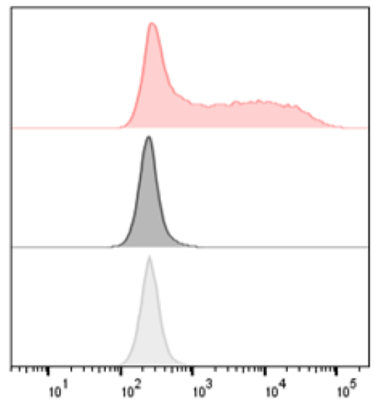

ACE2

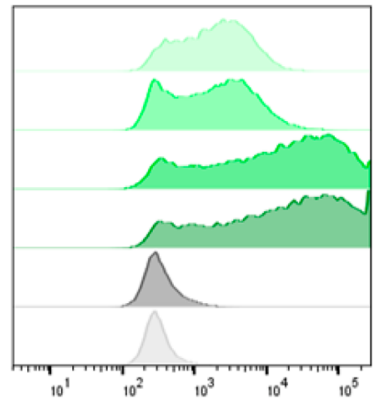

Human mAbs

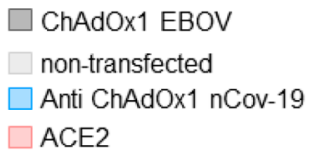

ChAdOx1 EBOV

Anti ChAdOx1 nCov-19

ACE2

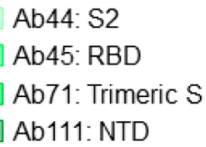

Ab44: S2

Ab71: Trimeric S

$\square$ Ab111: NTD

Figure 1. ChAdOxl nCoV-19 produces membrane associated SARS-CoV-2 S glycoprotein in native conformations able to bind its host receptor, ACE2. (A) Schematic representation of the vaccine encoded SARS-CoV-2 S protein, showing the position of N-linked glycosylation amino acid sequons $(\mathrm{NXS} / \mathrm{T}$, where $\mathrm{X} \neq \mathrm{P}$ ) as branches. Protein domains are illustrated: N-terminal domain (NTD), receptor-binding domain (RBD), fusion peptide (FP), heptad repeat 1 (HR1), central helix $(\mathrm{CH})$, connector domain (CD), and transmembrane domain (TM), with the additional tPA secretion signal at the N-terminus. (B) HeLa S3 cells were infected with ChAdOx1 nCoV-19 and incubated with recombinant ACE2, antiChAdOx1 nCoV-19 (derived from vaccinated mice), or a panel of human mAbs (Ab44, Ab45, Ab71, and Ab111, which recognize S2, RBD, trimeric S, and NTD, respectively) and compared to noninfected controls, analyzed by flow cytometry. (Left). Relative frequency of cells and AlexaFluor 488 fluorescence associated with antispike detection is plotted. Left, (blue) anti-ChAdOx1 nCoV-19; middle (red), ACE2; and right (shades of green) human mAbs. In dark gray cells infected with an irrelevant ChAdOx1 vaccine and in light gray noninfected cells are shown as a control. Experimental replicates were performed two times, and representative data are shown.

native-like functionality, prefusion trimeric structure, and glycosylation of SARS-CoV-2 S protein expressed from the ChAdOxl nCoV-19 vaccine.

\section{RESULTS AND DISCUSSION}

Expression of Prefusion Conformation SARS-CoV-2 S Glycoprotein on Cell Surfaces upon ChAdOx1 nCoV-19 Infection. ChAdOx1 nCoV-19 encodes a wild-type $S$ sequence, including the transmembrane domains, which is trafficked to the cell surface with a tissue plasminogen activator (tPA) signal sequence (Figure 1A). Using flow cytometry, we first detected the presence of the $S$ glycoprotein at the cell surface of ChAdOx1 nCoV-19 infected HeLa S3 cells (Figure 1B). Sera from mice vaccinated with ChAdOx1 nCoV-19 was used to detect the expression level of $S$ at the cell surface, revealing $\sim 60-70 \%$ of all cells expressing $S$ (range of duplicate averages across three experimental repeats) (Supplementary (Sup.) Figure 1). A ChAdOx1 vaccine encoding an irrelevant filovirus antigen (EBOV) was used as a negative control, $^{20}$ which showed a low level of binding to the antiChAdOx1 nCoV-19 vaccine serum $(\sim 0.5-2 \%$ cells across all experiments). This observation accounts for antibodies raised against the vector itself rather that the vaccine antigen. Subsequently, we sought to examine the properties of the cell surface expressed $S$ protein using recombinant ACE2 and a panel of human mAbs which bind to specific regions of $S$ (Figure 1B). Binding of infected cells to recombinant ACE2 confirms the correct folding of $S$ and native presentation and functionality of the RBD (Figure 1B). This observation is further supported by the binding of the human mAbs, in particular Ab45 which recognizes RBD, Ab71 which recognizes the trimeric spike, and Ab111 which recognizes the NTD. Ab44 which recognizes S2 also demonstrates considerable binding. These data confirm significant presence of the prefusion trimer at the cell surface. In the absence of a postfusion specific anti-S2 antibody we were unable to quantify if some postfusion spike is present at the cell surface.

While this observation that the majority of cells infected with ChAdOx1 nCoV-19 present native-like spikes on the cell surface (Figure 1B), it is interesting to note that a population may shed the S1 subunit. Whether this is a beneficial or detrimental feature with respect to the elicitation of immune responses during vaccination is unknown. Shedding of S1 subunits from viruses occurs during native infection, ${ }^{21-24}$ and the ChAdOx1 nCoV-19-derived $\mathrm{S}$ proteins mimic this native feature of the viral spike. Nevertheless it has been shown that vaccination with $\mathrm{ChAdOx} \mathrm{nCoV}-19$ elicits robust antibody and $\mathrm{T}$ cell responses. ${ }^{16-19}$

Structural Analysis of Membrane-Associated ChAdOx1 nCoV-19 Derived SARS-CoV-2 S Protein. After the 


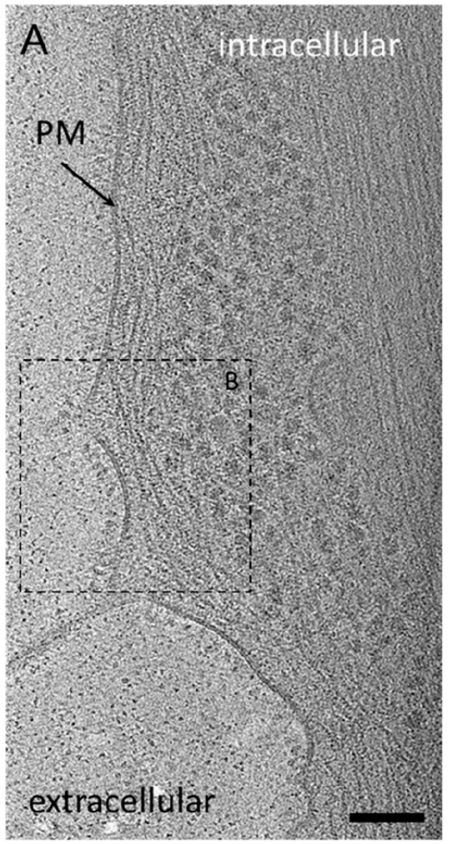

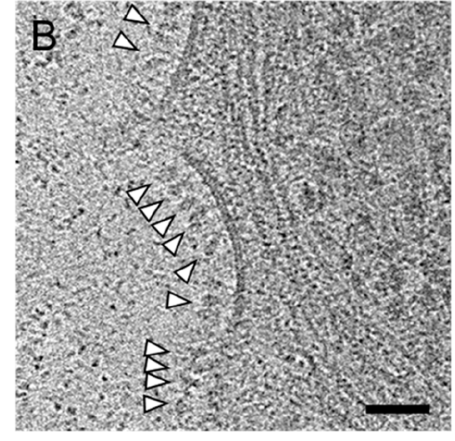

C

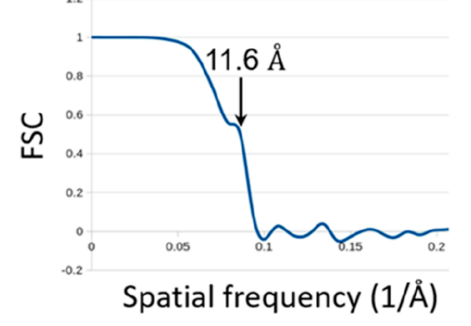

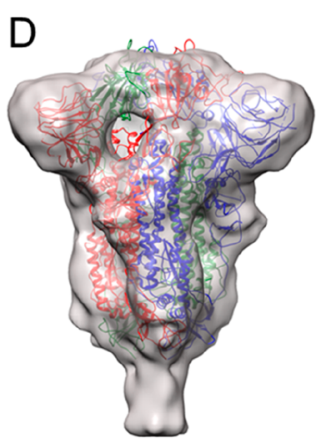

E

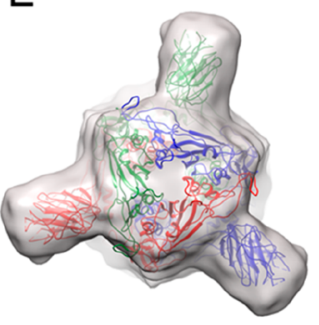

Figure 2. CryoET and subtomogram average of ChAdOx1 nCoV-19 derived spike. (A) Tomographic slice of U2OS cell transduced with ChAdOx1 $\mathrm{nCoV}-19$. The slice is $6.4 \AA$ thick; $\mathrm{PM}=$ plasma membrane, scale bar $=100 \mathrm{~nm}$. (B) Detailed view of the boxed area marked in (A). White arrowheads indicate spike proteins on the cell surface; scale bar $=50 \mathrm{~nm}$. (C-E) Subtomogram average of ChAdOx1 nCoV-19 spikes at $11.6 \AA$ resolution as indicated by Fourier-Shell correlation at 0.5 cutoff (C), shown from side view (D) and top view (E). SARS-CoV-2 atomic model $(\mathrm{PDB} 6 \mathrm{ZB} 5)^{29}$ is fitted for reference.

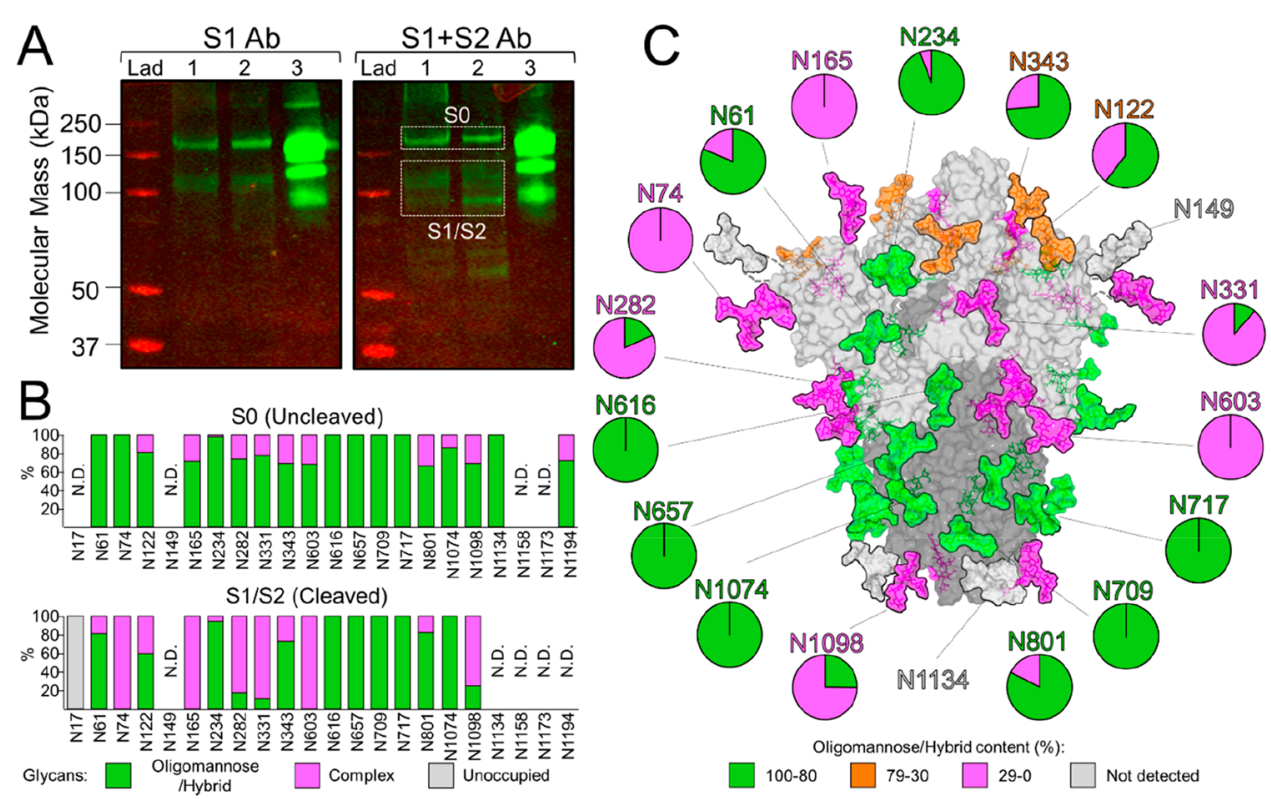

Figure 3. Site-specific glycan processing of SARS-CoV-2 S upon infection with ChAdOx1 nCoV-19. (A) Western blot analysis of SARS-CoV-2 spike proteins, using anti-S1 and anti-S1+S2 antibodies. Lane $1=$ Protein pellet from 293F cell lysates infected with ChAdOx1 nCoV-19. Lane $2=$ Reduced protein pellet from 293F infected with ChAdOx1 nCoV-19. Lane $3=2 \mathrm{P}$-stabilized SARS-CoV-2 S protein. The white boxes correspond to gel bands that were excised for mass spectrometric analysis. (B) Site-specific N-linked glycosylation of SARS-CoV-2 S0 and S1/S2 glycoproteins. The bar graphs represent the relative quantities of digested glycopeptides possessing the identifiers of oligomannose/hybrid-type glycans (green), complex-type glycans (pink), unoccupied PNGs (gray), or not determined (N.D.) at each N-linked glycan sequon on the $\mathrm{S}$ protein, listed from $\mathrm{N}$ to $\mathrm{C}$ terminus. (C) Glycosylated model of the cleaved (S1/S2) SARS-CoV-2 spike. The pie charts summarize the mass spectrometric analysis of the oligomannose/hybrid (green), complex (pink), or unoccupied (gray) N-linked glycan populations. Representative glycans are modeled onto the prefusion structure of trimeric SARS-CoV-2 S glycoprotein (PDB ID: 6VSB), with one RBD in the "up" conformation. The modeled glycans are colored according to oligomannose/hybrid-type glycan content with glycan sites labeled in green (80-100\%), orange (30-79\%), pink (0$29 \%)$, or gray (not detected).

surface expression of the $\mathrm{S}$ protein driven by ChAdOx1 $\mathrm{nCoV}$ 19 vector infection was confirmed by cytometry, we sought to probe the structure of $S$ proteins on native cell surfaces using cryo-electron tomography and subtomogram averaging (cry- 
oET STA). We imaged U2OS and HeLa cells infected with ChAdOxl nCoV-19. These cells were chosen for the thin cell peripheries which make them accessible for cryoET analysis. The tomograms revealed that the surface of the cells is densely covered with protruding densities consistent with the size and shape of the prefusion conformation of SARS-CoV-2 $S$ protein (Figure 2A and 2B, movie 1). These densities are absent in control uninfected cells (Sup. Figure 2). To determine whether these spikes represent the SARS-CoV-2 prefusion spike, we performed subtomogram averaging of 11391 spikes from cell surfaces using emClarity. ${ }^{25}$ The averaged density map, with 3fold symmetry applied, is at $11.6 \AA$ resolution (at 0.5 FSC cutoff) (Figure 2C), resolving the overall spike structure, which overlaps very well with prefusion spike atomic models in the literature ${ }^{4,26-29}$ (Figure 2D and 2E). We subsequently preformed cryo-immunolabeling using ChAdOx1 nCoV-19 vaccinated mice sera which confirmed the presentation of abundant $S$ protein on the cell surface, but not on control cells infected with ChAdOx-GFP or uninfected cells (Sup. Figure 3).

In order to investigate the presence of postfusion $S$ protein conformations, we employed template searching for pre- and postfusion spikes on cells infected with ChAdOx nCoV-19 (Sup. Figure 4). This analysis revealed a presence of abundant prefusion spikes on the cell surface, while little postfusion spikes are detected. Given the lack of a postfusion conformation specific anti-S2 antibody, we were not able to probe, by cryo-immunolabeling, postfusion spikes on the surface of cells.

The presentation of prefusion $S$ proteins was achieved through encoding SARS-CoV-2 S protein in the ChAdOx1 backbone without the incorporation of stabilizing mutations. Given that most neutralizing antibodies target epitopes displayed on the prefusion spike, the cryoET analysis revealing the expression of trimeric SARS-CoV-2 spike in the prefusion conformation strongly supports ChAdOx nCoV-19 as an effective vaccine strategy for the generation of a neutralizing immune response.

Site-Specific Glycan Analysis of the ChAdOx1 nCoV19 Derived SARS-CoV-2 S Protein Reveals Native-Like Glycan Maturation. The SARS-CoV-2 S gene encodes $22 \mathrm{~N}$ linked glycosylation sequons which span both the S1 and S2 subunits (Figure 1A). These host-derived glycans mask immunogenic protein epitopes from the humoral immune system, a common strategy utilized by viruses to evade the immune system. ${ }^{30}$ It is therefore of considerable importance that spike proteins produced upon vaccination successfully recapitulates the glycosylation observed on the virus, since antibodies may be elicited against protein epitopes that are occluded during natural infection. We sought to resolve the site-specific glycosylation of SARS-CoV-2 S proteins produced by cells following infection with ChAdOxl nCoV-19. To this end, human embryonic kidney 293 F (HEK293F) cells were incubated with ChAdOx1 nCoV-19. Western blot analysis of the protein pellets from the cell lysates using both S1- and S2specific antibodies confirmed the presence and maturation of the $S$ protein into the $S 1$ and $S 2$ subunits following furin cleavage. Since the whole cell was lysed, intracellular material of uncleaved S0 protein was also detected (Figure 3A). These gel bands were excised and analyzed by liquid chromatography-mass spectrometry (LC-MS) separately.

To determine the site-specific glycosylation and glycan occupancy of the spike protein, we employed trypsin, chymotrypsin, $\alpha$-lytic protease to generate glycopeptide samples. In order to increase the signal intensity of the glycopeptides, these samples were treated sequentially with Endoglycosidase $\mathrm{H}$ to cleave oligomannose- and hybrid-type glycans between the core GlcNAc residues, leaving a single GlcNAc attached to the Asn residue (+203 Da). Subsequent PNGase $\mathrm{F}$ treatment removed the remaining complex-type glycans which deamidates the Asn to Asp resulting in a $+3 \mathrm{Da}$ shift as the reaction was conducted in ${ }^{18} \mathrm{O}$ water. ${ }^{31-33}$ This homogenization of the glycans into oligomannose/hybrid-type and complex-type glycan categories was performed in order to increase coverage across the $S$ protein. The mass of peptides with unoccupied glycan sites exhibits no mass shift. The resulting peptide/glycopeptide pool from both the S1/S2 (cleaved) and So (uncleaved) proteins were subjected to LCMS analysis (Figure 3B).

The glycans presented on this S0 immature form of the protein were predominantly underprocessed oligomannose/ hybrid-type glycans (85\%) (Sup. Table 1), which reflects the intracellular origin of the protein, yet to be trafficked through to the cell membrane. This is consistent with furin protease being located in the trans-Golgi apparatus. In contrast, the cleaved S1/S2 protein, likely to be presented on the surface of cells, possesses significantly lower levels of these underprocessed glycans (56\%) and elevated levels of complex-type glycans (38\%). Furthermore, high levels of glycan occupancy were observed across the protein. This is especially important since glycans are known to shield more immunogenic protein epitopes; thus, the expression of SARS-CoV-2 S proteins using the ChAdOxl vaccine platform results in presentation of epitopes similar to those seen during natural infection. ${ }^{5,30,34}$ We note that the overall levels of oligomannose/hybrid-type glycans on the $\mathrm{S} 1 / \mathrm{S} 2$ protein were elevated compared to previous analyses; however, we attribute this to the lack of coverage of the glycosylation sites toward the C-terminus which are predominantly complex-type on recombinant proteins and viruses. ${ }^{5,27,35}$

Using the cryo-EM structure of the trimeric SARS-CoV-2 S protein, we mapped the glycosylation status of the S1/S2 protein (Figure 3C). A mixture of oligomannose/hybrid and complex-type sites were observed, with glycan sites such as $\mathrm{N} 234$, which are known to have stabilizing effects on the RBD, preserving the predominantly oligomannose state reported in both recombinant proteins and viruses. ${ }^{5,27,35,36}$ Similarly, the glycan at N165 which also stabilizes the RBD "up" conformation was determined to be complex-type on the $S$ protein arising from infection of cells with ChAdOx1 nCoV19. Since glycans are sensitive reporters of local protein architecture, it is encouraging that such glycans, known to have structural roles, conserve their processing state which provides additional evidence of native-like prefusion protein structure. Furthermore, there were no detectable traces of $\mathrm{O}$ linked glycans on the ChAdOxl-derived $S$ protein which may be due to their low abundances. This has been reflected in the low abundances of O-linked glycans from previous analysis on recombinant proteins, ${ }^{5,35}$ and lack of densities on EM maps. ${ }^{26}$ A site-specific comparison between previously analyzed stabilized recombinant $S$ protein analyses and the ChAdOx1 $\mathrm{nCoV}$-19-derived $\mathrm{S}$ protein is provided (Sup. Figure 5).

Adenoviruses have broad cell tropism due to the widespread distribution of their cellular receptors such as coxsackie and adenovirus receptor (CAR) and CD46. ${ }^{37,38}$ Replicationdeficient adenovirus vectored vaccines, such as ChAdOx 
$\mathrm{nCoV}-19$, are administered intramuscularly and predominantly induce antigen expression in muscle cells, fibroblasts, and professional antigen presenting cells (APCs, including dendritic cells) present in germinal centers of the lymph nodes. These APCs are primed by direct adenovirus infection or by cross-priming from nonimmune cells. ${ }^{38}$

During vaccination, SARS-CoV-2 S protein processing can be dependent on both the receptors present on and the enzymes expressed by the S-producing cells. ${ }^{39}$ Presence of the cell entry receptor ACE2 and host-cell proteases including furin and TMPRSS2 can drive conformational rearrangements resulting in postfusion conformations of $\mathrm{S}$. Tissue distribution of ACE2 is primarily in the nasal mucosa and GI tracts, and TMPRSS2 is predominantly expressed in lungs and GI tracts, where cells are susceptible to SARS-CoV-2 infection. In contrast, the muscle, fibroblast, and immune cell types targeted by intramuscularly administered ChAdOx1 nCoV-19 have not been shown to express high levels of ACE2 or TMPRSS2. ${ }^{40-42}$ The cell lines chosen for this study of the higher order structure and conformation vaccine-derived S (HeLa S3 and U20S) similarly do not express these enzymes in high abundance and are therefore a suitable surrogate for this in vitro study. Although HEK293F cells are not a natural host cell type in which the $S$ protein would be expressed upon administration of ChAdOx nCoV-19, due to the conservation of the enzymatic processing of the early stages of the mammalian N-linked glycosylation pathway, ${ }^{43,44}$ they represent a suitable model system to study the nature of the carbohydrate modifications of the $S$ protein. It may be useful in future to examine vaccine derived antigen processing in primary cells or even biopsy material, especially if alternative routes, such as intranasal administration, are considered.

Overall, this study provides holistic biophysical scrutiny of the spike protein that is produced upon vaccination with ChAdOxl nCoV-19. Importantly, we reveal the native-like mimicry of SARS-CoV-2 S protein's receptor binding functionality, prefusion structure, and processing of glycan modifications. While there is some evidence of $\mathrm{S} 1$ subunit shedding, as observed on the actual SARS-CoV-2 virus, the impact of the catabolism of the trimeric spike on immunogenicity and vaccine efficacy is yet to be determined. The data presented here will assist comparison across SARS$\mathrm{CoV}-2$ vaccination strategies and aid the development of nextgeneration immunogens.

\section{MATERIALS AND METHODS}

Production of ChAdOx1 nCoV-19. ChAdOx1 nCoV-19 was produced as described previously. ${ }^{17}$ The spike protein (S) of SARS-Cov-2 (Genbank accession number YP_009724390.1) was codon optimized for expression in human cell lines and synthesized by GeneArt Gene Synthesis (Thermo Fisher Scientific). The sequence encoding amino acids 2-1273 were cloned into a shuttle plasmid following InFusion cloning (Clontech). The shuttle plasmid encodes a modified human cytomegalovirus major immediate early promoter (IE CMV) with tetracycline operator (TetO) sites, a polyadenylation signal from bovine growth hormone (BGH), and a tPA signal sequence upstream of the inserted gene.

Production of ChAdOx1 nCoV-19 Derived SARS-CoV2 S. A liter of human embryonic kidney 293 Freestyle (HEK293F) cells, at $1 \times 10^{6}$ cells $/ \mathrm{ml}$ density, were infected with ChAdOx1 nCoV-19 at an MOI of $\sim 1$ viral particles per cell. The cells were incubated at $37^{\circ} \mathrm{C}$ for $48 \mathrm{~h}$ and pelleted in a centrifuge. The pellets were washed twice with phosphate buffered saline before addition of $5 \mathrm{~mL}$ of lysis buffer $(20 \mathrm{mM}$ Tris- $\mathrm{HCl}$ ( $\mathrm{pH} 8$ ), $150 \mathrm{mM} \mathrm{NaCl}, 0.5 \%$ IGEPAL, $0.25 \%$ sodium deoxycholate, $0.1 \%$ sodium dodecyl sulfate, $1 \mathrm{mM}$ EDTA) and sonication. The mixture was centrifuged at 4000 rpm for $30 \mathrm{~min}$, and the supernatant was collected. A $5 \mathrm{~mL}$ aliquot of lysis buffer and $3.5 \mathrm{~mL}$ of chloroform were added, and the tube was vortexed. $\mathrm{H}_{2} \mathrm{O}(9 \mathrm{~mL})$ was added, and after vortexing, the tube was centrifuged at $4000 \mathrm{rpm}$ for $30 \mathrm{~min}$. The upper liquid layer was removed, and $9 \mathrm{~mL}$ of methanol were added. After vortexing, the tube was centrifuged again. The methanol was subsequently removed, and the pellet was allowed to air-dry in the dark. Pellets were dissolved in $50 \mathrm{mM}$ sodium phosphate, $300 \mathrm{mM}$ sodium chloride, $\mathrm{pH} 7$.

Expression and Purification of Recombinant 2PStabilized SARS-CoV-2 S. Recombinant 2P-stabilized SARS-CoV-2 $\mathrm{S}$ protein was expressed as previously described. ${ }^{3,5}$ In brief, the expression vector containing the 2P-stabilized $S$ protein was used to transiently transfect FreeStyle293F cells. The protein was purified from the supernatant using nickel-affinity chromatography before sizeexclusion chromatography.

Western Blot Analysis. Protein pellets from the HEK293F cell lysates were loaded onto an SDS-PAGE gel and then transferred to a PVDF membrane. Polyclonal rabbit anti-S1 antibody (Sinobiological, Cat: 40592-T62) and anti-S2 antibody (Abcam, Cat: ab272504) were used as primary antibodies.

FACS Analysis for Cell Surface Spike Expression. Hela S3 cells at $4 \times 10^{5}$ cell $/ \mathrm{mL}$ were infected with 10 MOI ChAdOxl nCoV-19 and incubated at $37{ }^{\circ} \mathrm{C}$ for $40-48 \mathrm{~h}$. Cells were harvested and pelleted by centrifugation at $500 \mathrm{~g}$ for 5 min. Resuspended cells were split into three pools for the alternative staining and were incubated with either pooled prime-boost sera derived from outbred CD1 mice immunized intramuscularly with $10^{8}$ infectious units of ChAdOx1 nCoV$19,^{18}$ prepared at $1: 50$ in PBS-BSA-0.5\% or human mAbs prepared at $1 \mu \mathrm{g} / \mathrm{mL}$ or recombinant ACE2 expressed with a human Fc tag prepared at $2 \mu \mathrm{g} / \mathrm{mL}$ (see below). Cells were incubated for $2 \mathrm{~h}$ at rt. Cells were washed $3 \times$ with PBS-BSA$0.5 \%$ and then incubated for $1 \mathrm{~h}$ at $\mathrm{rt}$ with secondary antibodies conjugated with Alexafluor488 diluted at 1:1000 in PBS-BSA $0.5 \%$ (for ChAdOx1 nCov-19 serum staining Goat Anti mouse AlexaFluor 488 (Life Tech A11029), for ACE2 staining, Goat Anti Human AlexaFluor 488 (Life Tech)). Cells were washed twice with PBS-BSA-0.5\% before resuspending in PBS and analyzed by flow cytometry using a Fortessa X20 FACS analyzer. Samples were considered positive for spike expression if they had a fluorescence intensity above a threshold value determined by the maximum intensity of the noninfected control cells (Figure 1D). Experiments were performed twice in duplicate, and representative data are shown. Data were analyzed using FlowJo v9 (TreeStar).

ACE2 Expression. The ectodomain of the human angiotensin-converting enzyme 2 (ACE2) soluble construct encoding residues 18-740 (from NCBI Reference Sequence: NP_001358344.1.), where the transmembrane and cytoplasmic domains were removed, was expressed using human embryonic kidney $293 \mathrm{~F}$ cells (HEK293F). A N-terminal monoFc followed by TEV cleavage was included as well as a Cterminal four amino acid C-tag (EPEA) for affinity purification. ACE2 ectodomain was transiently expressed in Expi293 (Thermo Fisher Scientific) and protein purified from culture 
supernatants by C-tag affinity purification followed by gel filtration in Tris-buffered saline (TBS) $\mathrm{pH} 7.4$ buffer.

Isolation of Human Monoclonal Antibodies from Peripheral B Cells by Spike-Specific Single B Cells Sorting. To isolate Spike-specific B cells, PBMCs were labeled with recombinant trimeric spike-twin-Strep and stained with antibody cocktail consisting of CD3-FITC, CD14-FITC, CD56-FITC, CD16-FITC, IgM-FITC, IgA-FITC, IgD-FITC, IgG-BV786, CD19-BUV395, and Strep-MAB-DY549 (iba) to probe the Strep tag of spike. CD19+, IgG+, CD3-, CD14-, CD56-, CD16-, IgM-, IgA-, IgD-, Spike+ cells were then single cell sorted into 96-well PCR plates containing RNase inhibitor (N2611; Promega) and stored at $-80{ }^{\circ} \mathrm{C}$.

Genes encoding $\mathrm{Ig} \mathrm{VH}, \mathrm{Ig} \mathrm{V} \kappa$ and $\mathrm{V} \lambda$ from positive wells were amplified by RT-PCR (210210; QIAGEN) and nested PCR (203205; Qiagen) using 'cocktails' of primers specific for human IgG. PCR products of genes encoding heavy and light chains were joined with the expression vector for human IgG1 or Ig $\kappa$-chain or $\lambda$-chain (gifts from H. Wardemann) by Gibson assembly. Plasmids encoding heavy and light chains were then cotransfected into the 293T cell line by the polyethylenimine method (408727; Sigma), and antibodies were harvested for further study.

CryoET Sample Preparation and Imaging. EM grids (G300F1, R2/2 Quantifoil holey carbon, gold) were glowdischarged and treated with bovine fibronectin $(20 \mu \mathrm{g} / \mathrm{mL})$ for $30 \mathrm{~min}$. Grids were washed with PBS and UV-treated for $1 \mathrm{~h}$. $\mathrm{U} 2 \mathrm{OS}$ or HeLa cells $\left(1.6 \times 10^{5}\right)$ resuspended in $2 \mathrm{~mL}$ of DMEM 10\% FBS, Pen/Strep were seeded on top of the grids in 6-well plate wells. Cells and grids were incubated for $24 \mathrm{~h}$ at $37{ }^{\circ} \mathrm{C} / 5 \% \mathrm{CO}_{2}$ to allow cell attachment to grid carbon. ChAdOx1 nCoV-19 was added to the cells at MOI 1 , and cells were returned to $37{ }^{\circ} \mathrm{C} / 5 \% \mathrm{CO}_{2}$ for $48 \mathrm{~h}$. Grids were plungefrozen in liquid ethane at $-183{ }^{\circ} \mathrm{C}$ using the Leica GP2 plunger, after receiving $1 \mu \mathrm{L}$ of $5 \times$ concentrated $10 \mathrm{~nm} \mathrm{Au}$ fiducial solution (EMS) in the back-side and being blotted from the back. Grids were stored at liquid nitrogen until time of imaging.

Tilt series acquisition was carried out with an FEI Titan Krios G2 (Thermo Fisher Scientific) electron microscope operated at $300 \mathrm{kV}$ and equipped with a Gatan BioQuantum energy filter and post-GIF K3 detector (Gatan, Pleasanton, $\mathrm{CA}$ ) housed at electron Bioimaging Center (eBIC/Diamond Light Source, UK). Tilt series were acquired using SerialEM ${ }^{45}$ at a pixel size of $2.13 \AA$. Zero-loss imaging was used for all tilt series with a $20 \mathrm{eV}$ slit width. Defocus values ranged from -2 $\mu \mathrm{m}$ to $-7 \mu \mathrm{m}$.

A grouped dose-symmetric scheme was used for all tilt series, ${ }^{46}$ with a range of \pm 60 degrees at 3 degree increments in groups of 3 and total dose of $120-135 \mathrm{e}^{-} / \AA^{2}$. At each tilt, 5 movie frames were recorded using Correlated Double Sampling (CDS) in super-resolution mode and saved in lzw compressed tif format with no gain normalization. Movies were subsequently gain normalized during motion correction and Fourier cropped back to physical pixel size with MotionCor $2 .^{47}$

Tilt series were manually aligned using eTomo, ${ }^{48}$ and tilt series and alignment files were imported to emClarity. ${ }^{25} 11391$ subtomograms were selected after template matching using EMDB- $21452^{4}$ as the template, low-pass filtered to $40 \AA$. Subtomograms were separated in 2 completely independent half-data sets and iteratively aligned and averaged resulting in a final $11.6 \AA$ map at 0.5 FSC threshold. Model fitting and visualization were performed in Chimera. ${ }^{49}$
Glycopeptide Analysis by Liquid ChromatographyMass Spectrometry. Approximately 90 gel bands containing the furin cleaved (S1/S2) and uncleaved (S0) SARS-CoV-2 S protein were excised in order to gather sufficient material. The gel bands were destained in $50 \% \quad 100 \mathrm{mM}$ ammonium bicarbonate, $50 \%$ acetonitrile overnight. In-gel reduction and alkylation were performed according to the protocol by Shevchenko et al. ${ }^{50}$ The proteins were digested using trypsin, chymotrypsin, and $\alpha$-lytic protease, which cleave at the amino acids $\mathrm{R} / \mathrm{K}, \mathrm{F} / \mathrm{Y} / \mathrm{W}$, and $\mathrm{T} / \mathrm{A} / \mathrm{S} / \mathrm{V}$, respectively. After extraction, the peptide/glycopeptides were first treated with endoglycosidase $\mathrm{H}$ to deplete oligomannose-type glycans and leave a single GlcNAc residue at the corresponding site. Subsequently, the reaction mixture was completely dried and resuspended in a mixture containing PNGase F using only $\mathrm{H}_{2}{ }^{18} \mathrm{O}$ (Sigma-Aldrich) throughout. This reaction cleaves the remaining complex-type glycans but leaves the GlcNAc residues remaining after Endo $\mathrm{H}$ digestion intact. The use of $\mathrm{H}_{2}{ }^{18} \mathrm{O}$ enables complex-type glycan sites to be differentiated from unoccupied glycan sites since the hydrolysis of the glycosidic bond by PNGase $\mathrm{F}$ leaves an ${ }^{18} \mathrm{O}$ isotope on the resulting aspartic acid residue. The resulting peptides are purified using C18 Zip-tip (MerckMillipore) cleanup following the manufacturer's protocol. Eluted glycopeptides were dried and resuspended in $0.1 \%$ formic acid prior to analysis by liquid chromatography-mass spectrometry. An Easy-nLC 1200 (Thermo Fisher Scientific) system coupled to an Orbitrap Fusion mass spectrometer (Thermo Fisher Scientific) using higher energy collision-induced dissociation fragmentation was used. Peptides were separated using an EasySpray PepMap RSLC C18 column $(75 \mathrm{~cm} \times 75 \mu \mathrm{m})$ with a 275 min linear gradient consisting of $0-32 \%$ acetonitrile in $0.1 \%$ formic acid over $240 \mathrm{~min}$, followed by $35 \mathrm{~min}$ of $80 \%$ acetonitrile in $0.1 \%$ formic acid. The flow rate was set to $200 \mathrm{~nL} / \mathrm{min}$. The spray voltage was set to $2.7 \mathrm{kV}$, and the temperature of the heated capillary was set to $40{ }^{\circ} \mathrm{C}$. The ion transfer tube temperature was set to $275^{\circ} \mathrm{C}$. The scan range was $400-1600 \mathrm{~m} / z$. The HCD collision energy was set to $27 \%$. Precursor and fragment detection were performed at a resolution 100000 and 30000 for $\mathrm{MS}^{1}$ and $\mathrm{MS}^{2}$, respectively. The AGC target for $\mathrm{MS}^{1}$ and $\mathrm{MS}^{2}$ was $4 \mathrm{e}^{5}$ and $5 \mathrm{e}^{4}$, respectively, and the injection time for $\mathrm{MS}^{1}$ and $\mathrm{MS}^{2}$ was 50 and $54 \mathrm{~ms}$, respectively.

Glycopeptide fragmentation data were extracted from the raw file using Byonic and Byologic software (Version 3.5; Protein Metrics Inc.). The glycopeptide fragmentation data were evaluated manually for each glycopeptide; the peptide was scored as true-positive when the correct b-and y-fragment ions were observed. Two modifications were searched for + $203 \mathrm{Da}$ corresponding to a single GlcNAc residue, still attached to an asparagine residue of an N-linked glycan site, following the cleavage by Endo $\mathrm{H}$, and $+3 \mathrm{Da}$ corresponding to the ${ }^{18} \mathrm{O}$ deamidation product of a complex glycan. The relative quantities of each glycan type at each site as well as the unoccupied proportion were determined by comparison of the extracted ion chromatographic areas.

Glycosylated Model Construction. Structural models of $\mathrm{N}$-linked glycan presentation on SARS-CoV-2 S were created using electron microscopy structures (PDB ID 6VSB) along with complex-, hybrid-, and oligomannose-type N-linked glycans (PDB ID 4BYH, 4B7I, and 2WAH). A representative glycoform presented at each site was modeled on to the $\mathrm{N}$ linked carbohydrate attachment sites in Coot. ${ }^{51}$ 


\section{ASSOCIATED CONTENT}

\section{SI Supporting Information}

The Supporting Information is available free of charge at https://pubs.acs.org/doi/10.1021/acscentsci.1c00080.

Supplementary Figures $1-5$ and Table 1; FACS gating strategy, tomographic slices, cryo-EM images, sitespecific glycosylation comparisons between recombinant proteins, and values for glycoforms observed on $S$ proteins derived from ChAdOxl nCoV-19 (PDF)

Movie 1: Cryo-electron tomography of U2OS cells infected with ChAdOx1 nCoV-19 (MP4)

\section{AUTHOR INFORMATION}

\section{Corresponding Authors}

Max Crispin - School of Biological Sciences, University of Southampton, Southampton SO17 1BJ, U.K.; 이이.org/ 0000-0002-1072-2694; Email: max.crispin@soton.ac.uk

Peijun Zhang - Division of Structural Biology, Nuffield Department of Medicine, University of Oxford, Oxford OX3 $7 B N$, U.K.; Electron Bio-imaging Centre, Diamond Light Source, Didcot OX11 ODE, U.K.; 이이이.org/0000-00031803-691X; Email: peijun.zhang@strubi.ox.ac.uk

Sarah C. Gilbert - The Jenner Institute, Nuffield Department of Medicine, University of Oxford, Oxford, U.K.; NIHR Oxford Biomedical Research Centre, Oxford, U.K.; Email: sarah.gilbert@ndm.ox.ac.uk

Teresa Lambe - The Jenner Institute, Nuffield Department of Medicine, University of Oxford, Oxford, U.K.; NIHR Oxford Biomedical Research Centre, Oxford, U.K.;

Email: teresa.lambe@ndm.ox.ac.uk

\section{Authors}

Yasunori Watanabe - School of Biological Sciences, University of Southampton, Southampton SO17 1BJ, U.K.; Oxford Glycobiology Institute, Department of Biochemistry, University of Oxford, Oxford OX1 3QU, U.K.

Luiza Mendonça - Division of Structural Biology, Nuffield Department of Medicine, University of Oxford, Oxford OX3 $7 B N$, U.K.

Elizabeth R. Allen - The Jenner Institute, Nuffield Department of Medicine, University of Oxford, Oxford, U.K. Andrew Howe - Electron Bio-imaging Centre, Diamond Light Source, Didcot OX11 ODE, U.K.

Mercede Lee - The Wellcome Centre for Human Genetics, University of Oxford, Oxford OX3 7BN, U.K.

Joel D. Allen - School of Biological Sciences, University of Southampton, Southampton SO17 1BJ, U.K.; ㅇo이.org/ 0000-0003-2547-968X

Himanshi Chawla - School of Biological Sciences, University of Southampton, Southampton SO17 1BJ, U.K.

David Pulido - The Jenner Institute, Nuffield Department of Medicine, University of Oxford, Oxford, U.K.

Francesca Donnellan - The Jenner Institute, Nuffield Department of Medicine, University of Oxford, Oxford, U.K.

Hannah Davies - The Jenner Institute, Nuffield Department of Medicine, University of Oxford, Oxford, U.K.

Marta Ulaszewska - The Jenner Institute, Nuffield

Department of Medicine, University of Oxford, Oxford, U.K.

Sandra Belij-Rammerstorfer - The Jenner Institute, Nuffield Department of Medicine, University of Oxford, Oxford, U.K.; NIHR Oxford Biomedical Research Centre, Oxford, U.K.
Susan Morris - The Jenner Institute, Nuffield Department of Medicine, University of Oxford, Oxford, U.K.

Anna-Sophia Krebs - Division of Structural Biology, Nuffield Department of Medicine, University of Oxford, Oxford OX3 $7 B N$, U.K.

Wanwisa Dejnirattisai - The Wellcome Centre for Human Genetics, University of Oxford, Oxford OX3 7BN, U.K.

Juthathip Mongkolsapaya - The Wellcome Centre for Human Genetics and Chinese Academy of Medical Science (CAMS) Oxford Institute (COI), University of Oxford, Oxford OX3 7BN, U.K.; Dengue Hemorrhagic Fever Research Unit, Office for Research and Development, Faculty of Medicine, Siriraj Hospital, Mahidol University, Bangkok, Thailand

Piyada Supasa - The Wellcome Centre for Human Genetics, University of Oxford, Oxford OX3 7BN, U.K.

Gavin R. Screaton - The Wellcome Centre for Human Genetics and Division of Medical Sciences, John Radcliffe Hospital, University of Oxford, Oxford OX3 7BN, U.K.

Catherine M. Green - The Wellcome Centre for Human Genetics, University of Oxford, Oxford OX3 7BN, U.K.

Complete contact information is available at:

https://pubs.acs.org/10.1021/acscentsci.1c00080

\section{Author Contributions}

Y.W., L.M., and E.R.A. contributed equally to this work.

Author Contributions

Y.W., T.L, S.G., M.C., and P.Z. conceived the research and designed the experiments. L.M. prepared cell culture, cryoEM samples and with A.K. performed cryoET data processing and subtomogram averaging. A.H. collected cryoET data and performed initial tomography reconstruction. E.R.A. performed FACS experiments and analyzed data. D.P., F.D., H.D., and H.C. cloned, expressed and purified proteins in this work. G.R.S., J.M., W.D., and P.S. provided human monoclonal antibodies. Y.W. and J.A. performed mass spectrometry experiments and analyzed data. Y.W., L.M., E.R.A., T.L., S.G., M.C., and P.Z. analyzed data and wrote the paper. All authors commented on and approved the final manuscript.

\section{Notes}

The authors declare the following competing financial interest(s): Oxford University has entered into a partnership with AstraZeneca for further development of ChAdOx1 nCoV19. S.C.G. is co-founder of Vaccitech (collaborators in the early development of this vaccine candidate) and named as an inventor on a patent covering use of ChAdOxl-vectored vaccines and a patent application covering this SARS-CoV-2 vaccine. T.L. is named as an inventor on a patent application covering this SARS-CoV-2 vaccine and consultant to Vaccitech. Since initial submission of the manuscript, Y.W. has accepted a position at AstraZeneca; the design, experimental execution, and the write-up of this study were completed prior to this development.

Data and materials availability: The cryoEM density maps for ChAdOx nCoV-2019 was deposited in the EMDB under accession code EMD-12530. Mass spectrometry raw files have been deposited in the MassIVE proteomics database (MSV000086998).

\section{ACKNOWLEDGMENTS}

Early work on producing ChAdOxl $\mathrm{nCoV}-19$ was supported by Funding from the Department of Health and Social Care 
(DHSC) managed by the Engineering and Physical Sciences Research Council (EPSRC) for the Future Vaccine Manufacturing Research Hub Grant Reference: EP/R013756/1. This work was supported by the International AIDS Vaccine Initiative (IAVI) through Grant INV-008352/OPP1153692 and the IAVI Neutralizing Antibody Center through the Collaboration for AIDS Vaccine Discovery Grant OPP1196345/INV-008813, both funded by the Bill and Melinda Gates Foundation. This work was also supported by the National Institute for Allergy and Infectious Diseases through the Scripps Consortium for HIV Vaccine Development (CHAVD) (AI144462), the University of Southampton Coronavirus Response Fund, the Bright Future Trust, the National Institute for Allergy and Infectious Diseases Grant AI150481, and the UK Wellcome Trust Investigator Award 206422/Z/17/Z. We acknowledge Diamond for access and support of the CryoEM facilities at the UK national electron bioimaging centre (eBIC, Proposal NR18477, NR21005, and NT21004), funded by the Wellcome Trust, MRC and BBSRC. The computational aspects of this research were supported by the Wellcome Trust Core Award Grant Number 203141/Z/ $16 / Z$ and the NIHR Oxford BRC. C.G. is supported by Wellcome Award 090532/Z/09/Z. National Institute for Health Research Biomedical Research Centre Funding Scheme, the Chinese Academy of Medical Sciences (CAMS) Innovation Fund for Medical Science (CIFMS), China (Grant Number: 2018-I2M-2-002). G.R.S. is supported as a Wellcome Trust Senior Investigator (Grant 095541/A/11/Z).

\section{REFERENCES}

(1) Amanat, F.; Krammer, F. SARS-CoV-2 Vaccines: Status Report. Immunity. Cell Press: April 14, 2020; pp 583-589. DOI: 10.1016/ j.immuni.2020.03.007.

(2) Letko, M.; Marzi, A.; Munster, V. Functional Assessment of Cell Entry and Receptor Usage for SARS-CoV-2 and Other Lineage B Betacoronaviruses. Nat. Microbiol. 2020, 1-8.

(3) Wrapp, D.; Wang, N.; Corbett, K. S.; Goldsmith, J. A.; Hsieh, C.L.; Abiona, O.; Graham, B. S.; McLellan, J. S. Cryo-EM Structure of the 2019-NCoV Spike in the Prefusion Conformation. Science 2020, 367,1260 .

(4) Walls, A. C.; Park, Y. J.; Tortorici, M. A.; Wall, A.; McGuire, A. T.; Veesler, D. Structure, Function, and Antigenicity of the SARSCoV-2 Spike Glycoprotein. Cell 2020, 181 (2), 281-292 e6.

(5) Watanabe, Y.; Allen, J. D.; Wrapp, D.; McLellan, J. S.; Crispin, M. Site-Specific Glycan Analysis of the SARS-CoV-2 Spike. Science 2020, eabb9983.

(6) Krammer, F. SARS-CoV-2 Vaccines in Development. Nature 2020, 1-16.

(7) Polack, F. P.; Thomas, S. J.; Kitchin, N.; Absalon, J.; Gurtman, A.; Lockhart, S.; Perez, J. L.; Pérez Marc, G.; Moreira, E. D.; Zerbini, C.; et al. Safety and Efficacy of the BNT162b2MRNA Covid-19 Vaccine. N. Engl. J. Med. 2020, 383 (27), 2603-2615.

(8) Corbett, K. S.; Flynn, B.; Foulds, K. E.; Francica, J. R.; BoyogluBarnum, S.; Werner, A. P.; Flach, B.; O’Connell, S.; Bock, K. W.; Minai, M. Evaluation of the MRNA-1273 Vaccine against SARS-CoV2 in Nonhuman Primates. N. Engl. J. Med. 2020, 383, 1544.

(9) Gao, Q.; Bao, L.; Mao, H.; Wang, L.; Xu, K.; Yang, M.; Li, Y.; Zhu, L.; Wang, N.; Lv, Z.; et al. Development of an Inactivated Vaccine Candidate for SARS-CoV-2. Science 2020, 369 (6499), 7781.

(10) Liu, C.; Mendonæa, L.; Yang, Y.; Gao, Y.; Shen, C.; Liu, J.; Ni, T.; Ju, B.; Liu, C.; Tang, X. The Architecture of Inactivated SARSCoV-2 with Postfusion Spikes Revealed by Cryo-EM and Cryo-ET. Structure 2020, 28, 1218.

(11) Zost, S. J.; Gilchuk, P.; Case, J. B.; Binshtein, E.; Chen, R. E.; Nkolola, J. P.; Schäfer, A.; Reidy, J. X.; Trivette, A.; Nargi, R. S.; et al.
Potently Neutralizing and Protective Human Antibodies against SARS-CoV-2. Nature 2020, 584 (7821), 443-449.

(12) Robbiani, D. F.; Gaebler, C.; Muecksch, F.; Lorenzi, J. C. C.; Wang, Z.; Cho, A.; Agudelo, M.; Barnes, C. O.; Gazumyan, A.; Finkin, $\mathrm{S}$; et al. Convergent Antibody Responses to SARS-CoV-2 in Convalescent Individuals. Nature 2020, 584 (7821), 437-442.

(13) Du, S.; Cao, Y.; Zhu, Q.; Yu, P.; Qi, F.; Wang, G.; Du, X.; Bao, L.; Deng, W.; Zhu, H. Structurally Resolved SARS-CoV-2 Antibody Shows High Efficacy in Severely Infected Hamsters and Provides a Potent Cocktail Pairing Strategy. Cell 2020, 183, 1013.

(14) Barnes, C. O.; Jette, C. A.; Abernathy, M. E.; Dam, K.-M. A.; Esswein, S. R.; Gristick, H. B.; Malyutin, A. G.; Sharaf, N. G.; HueyTubman, K. E.; Lee, Y. E.; et al. SARS-CoV-2 Neutralizing Antibody Structures Inform Therapeutic Strategies. Nature 2020, 1-9.

(15) Brouwer, P. J. M.; Caniels, T. G.; van der Straten, K.; Snitselaar, J. L.; Aldon, Y.; Bangaru, S.; Torres, J. L.; Okba, N. M. A.; Claireaux, M.; Kerster, G.; et al. Potent Neutralizing Antibodies from COVID-19 Patients Define Multiple Targets of Vulnerability. Science 2020, No. eabc5902.

(16) Folegatti, P. M.; Ewer, K. J.; Aley, P. K.; Angus, B.; Becker, S.; Belij-Rammerstorfer, S.; Bellamy, D.; Bibi, S.; Bittaye, M.; Clutterbuck, E. A.; et al. Safety and Immunogenicity of the ChAdOx1 NCoV-19 Vaccine against SARS-CoV-2: A Preliminary Report of a Phase 1/2, Single-Blind, Randomised Controlled Trial. Lancet 2020, 0 (0). DOI: 10.1016/S0140-6736(20)31604-4.

(17) van Doremalen, N.; Lambe, T.; Spencer, A.; BelijRammerstorfer, S.; Purushotham, J. N.; Port, J. R.; Avanzato, V. A.; Bushmaker, T.; Flaxman, A.; Ulaszewska, M.; et al. ChAdOx1 NCoV19 Vaccine Prevents SARS-CoV-2 Pneumonia in Rhesus Macaques. Nature 2020, 1-5.

(18) Graham, S. P.; McLean, R. K.; Spencer, A. J.; BelijRammerstorfer, S.; Wright, D.; Ulaszewska, M.; Edwards, J. C.; Hayes, J. W. P.; Martini, V.; Thakur, N.; et al. Evaluation of the Immunogenicity of Prime-Boost Vaccination with the ReplicationDeficient Viral Vectored COVID-19 Vaccine Candidate ChAdOx1 NCoV-19. npj Vaccines 2020, 5 (1), 1-6.

(19) Ramasamy, M. N.; Minassian, A. M.; Ewer, K. J.; Flaxman, A. L.; Folegatti, P. M.; Owens, D. R.; Voysey, M.; Aley, P. K.; Angus, B.; Babbage, G.; et al. Safety and Immunogenicity of ChAdOx1 NCoV-19 Vaccine Administered in a Prime-Boost Regimen in Young and Old Adults (COV002): A Single-Blind, Randomised, Controlled, Phase 2/ 3 Trial. Lancet 2020, 0 (0). DOI: 10.1016/s0140-6736(20)32466-1.

(20) Sebastian, S.; Flaxman, A.; Cha, K. M.; Ulaszewska, M.; Gilbride, C.; Sharpe, H.; Wright, E.; Spencer, A. J.; Dowall, S.; Hewson, R.; et al. A Multi-Filovirus Vaccine Candidate: CoExpression of Ebola, Sudan, and Marburg Antigens in a Single Vector. Vaccines 2020, 8 (2). DOI: 10.3390/vaccines8020241.

(21) Zhang, L.; Jackson, C. B.; Mou, H.; Ojha, A.; Peng, H.; Quinlan, B. D.; Rangarajan, E. S.; Pan, A.; Vanderheiden, A.; Suthar, M. S.; et al. SARS-CoV-2 Spike-Protein D614G Mutation Increases Virion Spike Density and Infectivity. Nat. Commun. 2020, 11 (1), 19.

(22) Pallesen, J.; Wang, N.; Corbett, K. S.; Wrapp, D.; Kirchdoerfer, R. N.; Turner, H. L.; Cottrell, C. A.; Becker, M. M.; Wang, L.; Shi, W.; et al. Immunogenicity and Structures of a Rationally Designed Prefusion MERS-CoV Spike Antigen. Proc. Natl. Acad. Sci. U. S. A. 2017, 114 (35), E7348-E7357.

(23) Walls, A. C.; Tortorici, M. A.; Snijder, J.; Xiong, X.; Bosch, B. J.; Rey, F. A.; Veesler, D. Tectonic Conformational Changes of a Coronavirus Spike Glycoprotein Promote Membrane Fusion. Proc. Natl. Acad. Sci. U. S. A. 2017, 114 (42), 11157-11162.

(24) Berger, I.; Schaffitzel, C. The SARS-CoV-2 Spike Protein: Balancing Stability and Infectivity. Cell Research; Springer Nature: November 2, 2020; pp 1059-1060. DOI: 10.1038/s41422-02000430-4.

(25) Himes, B. A.; Zhang, P. EmClarity: Software for HighResolution Cryo-Electron Tomography and Subtomogram Averaging. Nat. Methods 2018, 15 (11), 955-961. 
(26) Turoňová, B.; Sikora, M.; Schürmann, C.; Hagen, W.; Welsch, S.; Blanc, F.; von Bülow, S.; Gecht, M.; Bagola, K.; Hörner, C.; et al. In Situ Structural Analysis of SARS-CoV-2 Spike Reveals Flexibility Mediated by Three Hinges. bioRxiv 2020, DOI: 10.1101/ 2020.06.26.173476.

(27) Yao, H.; Song, Y.; Chen, Y.; Wu, N.; Xu, J.; Sun, C.; Zhang, J.; Weng, T.; Zhang, Z.; Wu, Z. Molecular Architecture of the SARSCoV-2 Virus. Cell 2020, 183, 730.

(28) Ke, Z.; Oton, J.; Qu, K.; Cortese, M.; Zila, V.; McKeane, L.; Nakane, T.; Zivanov, J.; Neufeldt, C. J.; Cerikan, B.; et al. Structures and Distributions of SARS-CoV-2 Spike Proteins on Intact Virions. Nature 2020, 1-5.

(29) Toelzer, C.; Gupta, K.; Yadav, S. K. N.; Borucu, U.; Davidson, A. D.; Kavanagh Williamson, M.; Shoemark, D. K.; Garzoni, F.; Staufer, O.; Milligan, R.; et al. Free Fatty Acid Binding Pocket in the Locked Structure of SARS-CoV-2 Spike Protein. Science 2020, 370 (6517), 725-730.

(30) Watanabe, Y.; Bowden, T. A.; Wilson, I. A.; Crispin, M. Exploitation of Glycosylation in Enveloped Virus Pathobiology. Biochim. Biophys. Acta, Gen. Subj. 2019, 1863 (10), 1480-1497.

(31) Cao, L.; Pauthner, M.; Andrabi, R.; Rantalainen, K.; Berndsen, Z.; Diedrich, J. K.; Menis, S.; Sok, D.; Bastidas, R.; Park, S.-K. R.; et al. Differential Processing of HIV Envelope Glycans on the Virus and Soluble Recombinant Trimer. Nat. Commun. 2018, 9 (1), 3693.

(32) Cao, L.; Diedrich, J. K.; Kulp, D. W.; Pauthner, M.; He, L.; Park, S.-K. R.; Sok, D.; Su, C. Y.; Delahunty, C. M.; Menis, S.; et al. Global Site-Specific N-Glycosylation Analysis of HIV Envelope Glycoprotein. Nat. Commun. 2017, 8, 14954.

(33) Struwe, W. B.; Chertova, E.; Allen, J. D.; Seabright, G. E.; Watanabe, Y.; Harvey, D. J.; Medina-Ramirez, M.; Roser, J. D.; Smith, R.; Westcott, D.; et al. Site-Specific Glycosylation of Virion-Derived HIV-1 Env Is Mimicked by a Soluble Trimeric Immunogen. Cell Rep. 2018, 24 (8), 1958-1966 e5.

(34) Watanabe, Y.; Berndsen, Z. T.; Raghwani, J.; Seabright, G. E.; Allen, J. D.; Pybus, O. G.; McLellan, J. S.; Wilson, I. A.; Bowden, T. A.; Ward, A. B.; et al. Vulnerabilities in Coronavirus Glycan Shields despite Extensive Glycosylation. Nat. Commun. 2020, 11 (1), 2688.

(35) Zhao, P.; Praissman, J. L.; Grant, O. C.; Chen, B.; Brief, I.; Cai, Y.; Xiao, T.; Rosenbalm, K. E.; Aoki, K.; Kellman, B. P. VirusReceptor Interactions of Glycosylated SARS-CoV-2 Spike and Human ACE2 Receptor. Cell Host Microbe 2020, 28, 1-16.

(36) Brun, J.; Vasiljevic, S.; Gangadharan, B.; Hensen, M.; Chandran, A. V.; Hill, M. L.; Kiappes, J. L.; Dwek, R. A.; Alonzi, D. S.; Struwe, W. B.; et al. Analysis of SARS-CoV-2 Spike Glycosylation Reveals Shedding of a Vaccine Candidate. bioRxiv. bioRxiv November 16, 2020; DOI: 10.1101/2020.11.16.384594.

(37) Morris, S. J.; Sebastian, S.; Spencer, A. J.; Gilbert, S. C. Simian Adenoviruses as Vaccine Vectors. Future Virology; Future Medicine Ltd: September 1, 2016; pp 649-659. DOI: 10.2217/fvl-2016-0070.

(38) Dicks, M. D. J.; Spencer, A. J.; Coughlan, L.; Bauza, K.; Gilbert, S. C.; Hill, A. V. S.; Cottingham, M. G. Differential Immunogenicity between HAdV-5 and Chimpanzee Adenovirus Vector ChAdOx1 Is Independent of Fiber and Penton RGD Loop Sequences in Mice. Sci. Rep. 2015, 5 (1), 1-15.

(39) Essalmani, R.; Jain, J.; Susan-Resiga, D.; Andréo, U.; Evagelidis, A.; Derbali, R. M.; Huynh, D. N.; Dallaire, F.; Laporte, M.; Delpal, A.; et al. Furin Cleaves SARS-CoV-2 Spike-Glycoprotein at S1/S2 and S2' for Viral Fusion/Entry: Indirect Role of TMPRSS2. bioRxiv 2020, DOI: $10.1101 / 2020.12 .18 .423106$.

(40) Uhlen, M.; Fagerberg, L.; Hallstrom, B. M.; Lindskog, C.; Oksvold, P.; Mardinoglu, A.; Sivertsson, A.; Kampf, C.; Sjostedt, E.; Asplund, A.; et al. Tissue-Based Map of the Human Proteome. Science 2015, 347 (6220), 1260419-1260419.

(41) Hamming, I.; Timens, W.; Bulthuis, M. L. C.; Lely, A. T.; Navis, G. J.; van Goor, H. Tissue Distribution of ACE2 Protein, the Functional Receptor for SARS Coronavirus. A First Step in Understanding SARS Pathogenesis. J. Pathol. 2004, 203 (2), 631637.
(42) Sungnak, W.; Huang, N.; Bécavin, C.; Berg, M.; Queen, R.; Litvinukova, M.; Talavera-López, C.; Maatz, H.; Reichart, D.; Sampaziotis, F.; et al. SARS-CoV-2 Entry Factors Are Highly Expressed in Nasal Epithelial Cells Together with Innate Immune Genes. Nat. Med. 2020, 26 (5), 681-687.

(43) Kornfeld, R.; Kornfeld, S. Assembly of Asparagine-Linked Oligosaccharides. Annu. Rev. Biochem. 1985, 54 (1), 631-664.

(44) Gagneux, P.; Aebi, M.; Varki, A. Evolution of Glycan Diversity; Cold Spring Harbor Laboratory Press: 2015. DOI: 10.1101/ GLYCOBIOLOGY.3E.020.

(45) Mastronarde, D. N. Automated Electron Microscope Tomography Using Robust Prediction of Specimen Movements. J. Struct. Biol. 2005, 152 (1), 36-51.

(46) Hagen, W. J. H.; Wan, W.; Briggs, J. A. G. Implementation of a Cryo-Electron Tomography Tilt-Scheme Optimized for High Resolution Subtomogram Averaging. J. Struct. Biol. 2017, 197 (2), 191-198.

(47) Zheng, S. Q.; Palovcak, E.; Armache, J. P.; Verba, K. A.; Cheng, Y.; Agard, D. A. MotionCor2: Anisotropic Correction of BeamInduced Motion for Improved Cryo-Electron Microscopy. Nat. Methods 2017, 14 (4), 331-332.

(48) Kremer, J. R.; Mastronarde, D. N.; McIntosh, J. R. Computer Visualization of Three-Dimensional Image Data Using IMOD. J. Struct. Biol. 1996, 116 (1), 71-76.

(49) Pettersen, E. F.; Goddard, T. D.; Huang, C. C.; Couch, G. S.; Greenblatt, D. M.; Meng, E. C.; Ferrin, T. E. UCSF Chimera - A Visualization System for Exploratory Research and Analysis. J. Comput. Chem. 2004, 25 (13), 1605-1612.

(50) Shevchenko, A.; Tomas, H.; Havli, J.; Olsen, J. V.; Mann, M. In-Gel Digestion for Mass Spectrometric Characterization of Proteins and Proteomes. Nat. Protoc. 2006, 1 (6), 2856-2860.

(51) Emsley, P.; Crispin, M. Structural Analysis of Glycoproteins: Building N-Linked Glycans with Coot. Acta Crystallogr. Sect. D Struct. Biol. 2018, 74 (4), 256-263. 